\title{
Salivary Gland Disease in the Era of COVID-19 Pandemic
}

\author{
Liuba Soldatova et al. ${ }^{1}$ \\ ${ }^{1}$ Affiliation not available
}

April 28, 2020

\section{Introduction}

The rapid spread of coronavirus disease 2019 (COVID-19) worldwide raised concerns about its heavy impact on the health care delivery system and forced significant changes in the realities of the clinical practice we are accustomed to. With these changes comes a need for a different approach to outpatient evaluation of common otolaryngology complaints in patients with new symptoms.

Recently published set of guidelines for evaluation of head and neck during the COVID-19 pandemic recommended to postpone the management of benign disease including benign salivary or thyroid gland disease. ${ }^{1}$ In order to limit the chance of COVID-19 infection among patients or health care workers, surveying patients via telephone or telemedicine visit was advised, reserving in-person evaluation for the patients at risk for significant negative outcomes. The challenge is that these measures can only be applied in clear-cut clinical scenarios, when the disease process is most likely benign and the care delivery can be postponed.

In cases with a high degree of uncertainty based on available clinical information, many physicians will have to decide how to proceed after initial telemedicine encounter. Clinicians will have to consider how to balance a potential delay in diagnosis, including cancer diagnosis, against the risk of COVID-19 exposure, and may need to exercise their best judgement knowing that for head and neck cancer the risk of progression with cancer care delay is high. ${ }^{2}$ In this communication, we present our approach to triaging and evaluation of patients with complaints concerning for salivary gland disease.

\section{Initial Encounter}

New patients with complaints of swelling and/or pain in the area of major salivary glands should be interviewed and visually examined during the initial telemedicine encounter. To compensate for the limitations of the visual exam, patients should be instructed to palpate the area of the affected salivary gland to determine the presence of a palpable mass. Although this maneuver might not to be trivial or reliable, self-palpation should be attempted as a screening method since a large portion of head and neck masses can be detected on self-exam.

Patients should be asked about the major causes of inflammatory salivary gland disease such as history of salivary gland stones, auto-immune conditions, history of receiving iodine contrast, radioactive iodine or radiation to the head and neck area. ${ }^{3}$ History of skin malignancies of the head and neck and history of other cancers should be taken into account as a potential cause for metastatic lesions within the salivary glands (Table 1).

A thorough description of presenting symptoms must be determined since inflammatory and neoplastic salivary gland disease have different presentations. Special emphasis should be placed on chronicity of symptoms and any red flag symptoms such as a palpable mass, facial muscle weakness, and reports of paresthesia (Table 1). If the onset of salivary gland swelling and pain is gradual and progressive, and 
the patient reports palpating a mass in the area of salivary gland, workup should be initiated to provide additional information and rule out salivary gland neoplasm.., 5

If the symptoms are consistent with inflammatory or infectious sialadenitis without a palpable mass, medical management should be initiated and a follow-up telemedicine visit should be scheduled to ensure symptom resolution. If the symptoms persist or relapse after appropriate medical management, imaging should be considered, since obstructive salivary gland disease such as sialadenitis could be caused by the salivary gland neoplasm or coexist with salivary gland neoplasm and metastatic lesions within the salivary gland (Figure $1) .^{6}$

\section{Diagnostic Workup}

After initial prescreening during the telemedicine encounter, patients with signs or symptoms of a salivary gland mass should undergo diagnostic imaging. All patients must be screened for symptoms of COVID-19 or risk factors for exposure prior to their visit to the radiology department. Imaging studies of the patients with the symptoms concerning for COVID-19 should be cancelled and delayed until the diagnosis is confirmed and they recover from their illness, or COVID-19 is ruled out with negative test results unless this delay has a high probability of significant negative outcome for the patient. ${ }^{7}$ The most up-to-date institutional guidelines should be used to determine who needs COVID-19 testing. If possible, patients must undergo imaging at the sites with less foot traffic and with fewer critically ill patients to prevent secondary exposure to COVID-19.

The goal of radiographic evaluation is to assist in the triage of patients with a suspected salivary gland mass and to determine if the patient has a lesion requiring a tissue diagnosis for treatment recommendations. The most readily available imaging studies must be utilized with the preference given to ultrasonographic evaluation due to ease of performance and the ability to immediately obtain a fine-needle aspiration biopsy (FNAB) of identified lesions for cytopathologic analysis (Table 1). Since several benign and malignant salivary gland lesions can present with minor or predominant cystic component, and one third of cystic salivary gland lesions are neoplastic, ${ }^{8}$ presence of a cystic component on ultrasonography should not serve as a determining factor in the decision to proceed with FNAB. Salivary gland ultrasound can also aid in the diagnosis of sialolithiasis or other inflammatory salivary gland conditions, and guide further treatment recommendations. ${ }^{9,10}$ If FNAB cannot be done, consideration should be given to contrast-enhanced magnetic resonance imaging (MRI) instead of the ultrasound to confirm presence of a salivary gland mass and to look for radiographic features of common benign or malignant salivary gland tumors.

Clinical situations of radiographically diagnosed salivary gland masses, especially deep parotid lobe masses not amenable to FNAB must be approached on a case-by-case basis with diagnostic options of either computed tomography (CT) guided biopsy or surgical resection. The final diagnostic choice will depend on the availability of the hospital's interventional radiology department and COVID-19 burden. For patients with minor salivary gland tumors of the oral cavity, sinonasal region and pharynx diagnosed based on the office-based biopsy, appropriate cross-sectional imaging of the head and neck should be performed upfront to determine the extent of the lesion and the treatment plan.

Telemedicine visits can be used to determine the best location for salivary gland imaging. Good knowledge of local private imaging facilities willing to perform salivary gland ultrasound, FNAB or other head and neck imaging when the hospitals with high volume of COVID-19 patients postpone it will help determine best options for radiology referrals. Fortunately, evaluation of major salivary gland neoplasms can be done without oral or nasal examination, and some otolaryngology physicians have the capabilities to perform an ultrasound and an FNAB in the office. After completion of salivary gland ultrasound and FNAB, MRI with contrast must be reserved for preoperative planning in patients with time-sensitive diagnosis such as salivary gland malignancy and can be ordered on the basis of the US and the FNAB results if indicated. Imaging of sialolithiasis and other inflammatory salivary gland conditions (CT or sialo-MRI) should be postponed in the majority of patients. However, in rare situations such imaging might become unavoidable (Figure 1).

Ultrasound characteristics alone cannot distinguish benign from malignant salivary gland masses, and must 
be considered in conjunction with the FNAB results. ${ }^{11,12}$ FNAB results can be stratified by the Milan System for Reporting Salivary Gland Cytology (MSRSGC) ${ }^{13-14}$ to render treatment recommendations. Repeat FNAB must be considered if the initial FNAB results are interpreted as "non-diagnostic" (Table 2). Personal protective equipment (PPE) should be used appropriately while performing FNAB procedures and handling specimens according to the College of American Pathologist guidelines. ${ }^{15}$ Only essential members of the health care team should be present during the FNAB procedure to reduce exposure as expelling aspirates may generate droplets or aerosols. In addition, agitating air-dried smears should be minimized.

\section{Patient Counselling and Treatment Recommendations}

Patients with the FNAB results concerning for salivary gland malignancy and patients with confirmed salivary gland malignancies diagnosed after an office-based or CT-guided biopsy should be counselled about the prognosis and the need for surgical management despite of the surgical case delays due to COVID-19 pandemic. It is important to discuss with the patient the fact that salivary gland FNAB interpretation based on MSRSGC has excellent, but variable reported sensitivity (86-100\%) and specificity (90-100\%) in differentiating between benign and malignant lesions. ${ }^{16}$ Unless the FNAB results are categorized as "non-neoplastic" based on MSRSGC (Table 2), ${ }^{14}$ surgical management should be discussed, while serious considerations are given to higher risk of contracting COVID-19 and related complications in cancer patients (Table 1). ${ }^{13,14}$

The current prevalence of COVID-19 positive patients in the geographic area and within the hospital must be taken into account when discussing surgery. Prior to any surgical intervention, patients should ideally undergo COVID-19 testing. Clinicians must be aware of false negatives with COVID-19 testing, and the fact that repeat testing for COVID-19 is not feasible in most patients. Chest CT may be considered in select cases for further evaluation and risk stratification to assess for signs of COVID-19 related pulmonary involvement or metastases. ${ }^{17}$ Surgery should be postponed for all patients with suspicious chest CT findings, those with symptoms of COVID-19 or positive COVID-19 test results.

When surgery is deemed necessary, surgical teams need to consider the risk of aerosolization. For minor salivary gland tumors of the oral cavity, sinonasal region and pharynx, appropriate PPE of the entire operating room staff is highly recommended. Operating room personnel must be minimized. For parotid and submandibular gland neoplasm surgeries, the risk of aerosolized mucosal secretions is only encountered during intubation and extubation. All non-essential personnel should leave the room during intubation and extubation for these surgeries, with appropriate PPE worn by all staff present in the room. ${ }^{1}$

Management of obstructive salivary gland disease should be carried out remotely in most cases. In rare cases, office-based evaluation and treatment interventions or an intraoperative drainage and biopsy may be required to rule out cancer, to manage an abscess or to relieve severe pain not controlled after appropriate medical management. Sialendoscopy should be avoided during the COVID-19 pandemic, given the irrigation and the aerosolization.

\section{Conclusion}

Current COVID-19 pandemic with a need to preserve health care resources and staff can result in cancer care delays and negative impact on patient outcomes. Salivary gland swelling and pain are commonly associated with benign pathology, but the risk of salivary gland malignancy should not be overlooked in the subset of patients with the history and initial evaluation concerning for salivary gland mass. We believe that these patients should undergo additional diagnostic workup to avoid delays in cancer care and negative impact on outcomes. Patient triage, ultrasonographic evaluation and FNAB of salivary gland lesions or, in some cases, immediate contrast-enhanced MRI can help avoid some of the delays. Additional cross-sectional imaging and diagnostic procedures must be postponed if possible. All measures must be taken to minimize COVID-19 exposure.

\section{References}

1. Givi B, Schiff BA, Chinn SB, et al. Safety Recommendations for Evaluation and Surgery of the Head and Neck During the COVID-19 Pandemic. JAMA Otolaryngol Neck Surg. March 2020. 
2. Kutikov A, Weinberg DS, Edelman MJ, Horwitz EM, Uzzo RG, Fisher RI. A War on Two Fronts: Cancer Care in the Time of COVID-19. Ann Intern Med . March 2020.

3. Harrison JD. Causes, Natural History, and Incidence of Salivary Stones and Obstructions. Otolaryngol Clin North Am . 2009;42(6):927-947.

4. Lewis AG, Tong T, Maghami E. Diagnosis and Management of Malignant Salivary Gland Tumors of the Parotid Gland. Otolaryngol Clin North Am . 2016;49(2):343-380.

5. Mantravadi AV, Moore MG, Rassekh CH. AHNS series: Do you know your guidelines? Diagnosis and management of salivary gland tumors. Head Neck . 2019;41(2):269-280.

6. Witt RL. Major salivary gland cancer. Surg Oncol Clin N Am . 2004;13(1):113-127.

7. Mossa-Basha M, Meltzer CC, Kim DC, Tuite MJ, Kolli KP, Tan BS. Radiology Department Preparedness for COVID-19: Radiology Scientific Expert Panel. Radiology . March 2020:200988.

8. Pantanowitz L, Thompson LDR, Rossi ED. Diagnostic Approach to Fine Needle Aspirations of Cystic Lesions of the Salivary Gland. Head Neck Pathol . 2018;12(4):548-561.

9. Katz P, Hartl DM, Guerre A. Clinical Ultrasound of the Salivary Glands. Otolaryngol Clin North Am . 2009;42(6):973-1000.

10. Yariv O, Popovtzer A, Wasserzug O, et al. Usefulness of ultrasound and fine needle aspiration cytology of major salivary gland lesions. Am J Otolaryngol . 2020;41(1):102293.

11. Wu S, Liu G, Chen R, Guan Y. Role of ultrasound in the assessment of benignity and malignancy of parotid masses. Dentomaxillofacial Radiol . 2012;41(2):131-135.

12. Rzepakowska A, Osuch-Wójcikiewicz E, Sobol M, Cruz R, Sielska-Badurek E, Niemczyk K. The differential diagnosis of parotid gland tumors with high-resolution ultrasound in otolaryngological practice. Eur Arch Otorhinolaryngol . 2017;274(8):3231-3240.

13. Rossi ED, Faquin WC, Baloch Z, et al. The Milan System for Reporting Salivary Gland Cytopathology: Analysis and suggestions of initial survey. Cancer Cytopathol . 2017;125(10):757-766.

14. Barbarite E, Puram SV, Derakhshan A, Rossi ED, Faquin WC, Varvares MA. A Call for Universal Acceptance of the Milan System for Reporting Salivary Gland Cytopathology. The Laryngoscope . 2020;130(1):8085.

15. Cytopathology Laboratory Considerations During the COVID-19 Pandemic. College of American Pathologists. https://www.cap.org/laboratory-improvement/news-and-updates/cytopathology-laboratory-considerationsduring-the-covid-19-pandemic. Accessed April 8, 2020.

16. Kala C, Kala S, Khan L. Milan System for Reporting Salivary Gland Cytopathology: An Experience with the Implication for Risk of Malignancy. J Cytol . 2019;36(3):160-164.

17. Li Y, Xia L. Coronavirus Disease 2019 (COVID-19): Role of Chest CT in Diagnosis and Management. Am J Roentgenol . March 2020:1-7.

Table 1. Evaluation of Patients with Salivary Gland Disease.

Initial Telemedicine Encounter

Identify patients with: palpable or visually identifiable mass in the area of major salivary gland symptoms concerning for $n$ Diagnostic Workup of Patients with Suspected Salivary Gland Neoplasm

Screen for COVID-19 Salivary gland ultrasound FNA biopsy of neoplastic lesions Additional imaging if needed for surgical Patient Counselling and Treatment Recommendations

Discuss FNA biopsy results Review treatment and prognosis COVID-19 testing if surgery is anticipated Referrals to Radiat 
Table 2. The Milan Classification of Salivary Gland Cytopathology. ${ }^{13-14}$

\begin{tabular}{lll}
\hline Diagnostic Category & Risk of Malignancy (\%) & Management* \\
\hline Nondiagnostic & 25 & Repeat FNA, cli \\
Non-neoplastic & 10 & Clinical follow-u \\
Atypia of undetermined significance & 20 & Repeat FNA or s \\
Neoplasm: benign & $<5$ & Surgery or clinic \\
Neoplasm: salivary gland neoplasm of uncertain malignant potential (SUMP) & 35 & Surgery \\
Suspicious for malignancy & 60 & Surgery \\
Malignant & $>90$ & Surgery \\
\hline
\end{tabular}

*Actual management may depend on the results of follow-up and imaging
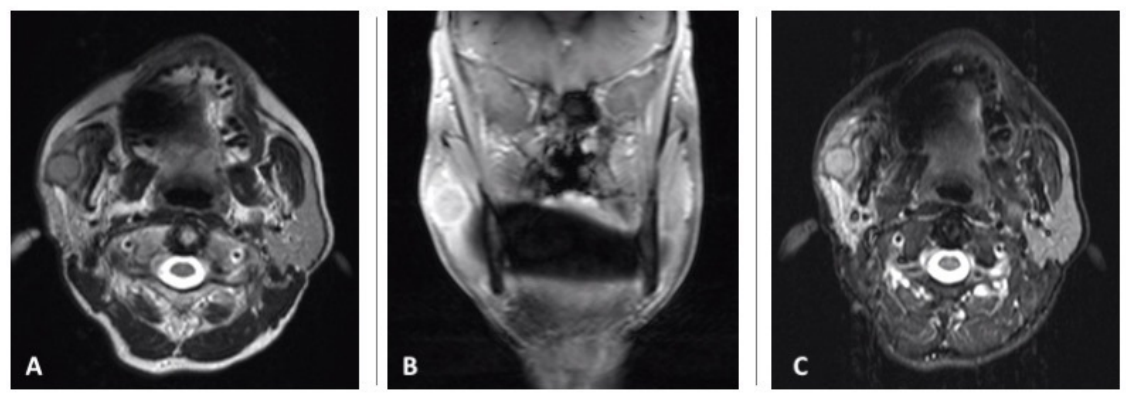

Figure 1. Gadolinium enhanced head and neck MRI of a 65-year-old female with long-standing recurrent right parotid gland swelling and pain thought to be caused by sialolithiasis with FNAB positive for p16(+) squamous cell carcinoma. (A) axial STIR (B) T1 weighted coronal and (C) T2 weighted axial images of the right parotid gland mass $15.5 \mathrm{~mm}$ in largest dimension. 